University of Wollongong

Research Online

Australian Institute for Innovative Materials -

Papers

Australian Institute for Innovative Materials

$1-1-2013$

Fusion of nacre, mussel, and lotus leaf: bio-inspired graphene composite paper with multifunctional integration

\author{
Da Zhong \\ Beihang University \\ Qinglin Yang \\ Beihang University \\ Lin Guo \\ Beihang University \\ SX. Dou \\ University of Wollongong, shi@uow.edu.au \\ Kesong Liu \\ University of Wollongong
}

See next page for additional authors

Follow this and additional works at: https://ro.uow.edu.au/aiimpapers

Part of the Engineering Commons, and the Physical Sciences and Mathematics Commons

Research Online is the open access institutional repository for the University of Wollongong. For further information contact the UOW Library: research-pubs@uow.edu.au 


\title{
Fusion of nacre, mussel, and lotus leaf: bio-inspired graphene composite paper with multifunctional integration
}

\author{
Abstract \\ Multifunctional integration is an inherent characteristic for biological materials with multiscale structures. \\ Learning from nature is an effective approach for scientists and engineers to construct multifunctional \\ materials. In nature, mollusks (abalone), mussels, and the lotus have evolved different and optimized \\ solutions to survive. Here, bio-inspired multifunctional graphene composite paper was fabricated in situ \\ through the fusion of the different biological solutions from nacre (brick-and-mortar structure), mussel \\ adhesive protein (adhesive property and reducing character), and the lotus leaf (self-cleaning effect). \\ Owing to the special properties (self-polymerization, reduction, and adhesion), dopamine could be \\ simultaneously used as a reducing agent for graphene oxide and as an adhesive, similar to the mortar in \\ nacre, to crosslink the adjacent graphene. The resultant nacre-like graphene paper exhibited stable \\ superhydrophobicity, self-cleaning, anti-corrosion, and remarkable mechanical properties underwater.
}

\section{Keywords}

mussel, lotus, fusion, graphene, leaf, composite, bio, paper, nacre, multifunctional, inspired, integration

Disciplines

Engineering | Physical Sciences and Mathematics

\section{Publication Details}

Zhong, D., Yang, Q., Guo, L., Dou, S. Xue., Liu, K. \& Jiang, L. (2013). Fusion of nacre, mussel, and lotus leaf: bio-inspired graphene composite paper with multifunctional integration. Nanoscale, 5 (13), 5758-5764.

\section{Authors}

Da Zhong, Qinglin Yang, Lin Guo, S X. Dou, Kesong Liu, and Lei Jiang 


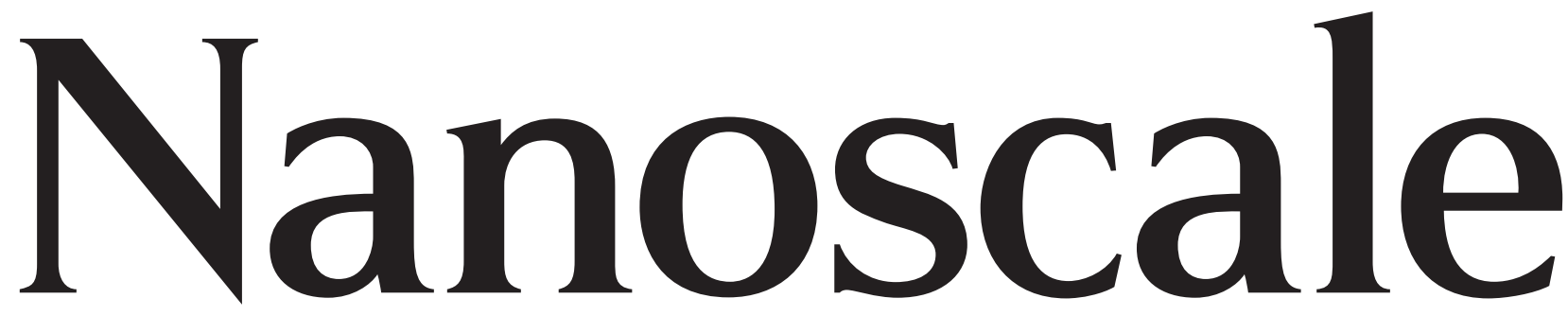

www.rsc.org/nanoscale

Volume 5 | Number 13 | 7 July 2013 | Pages 5657-6188

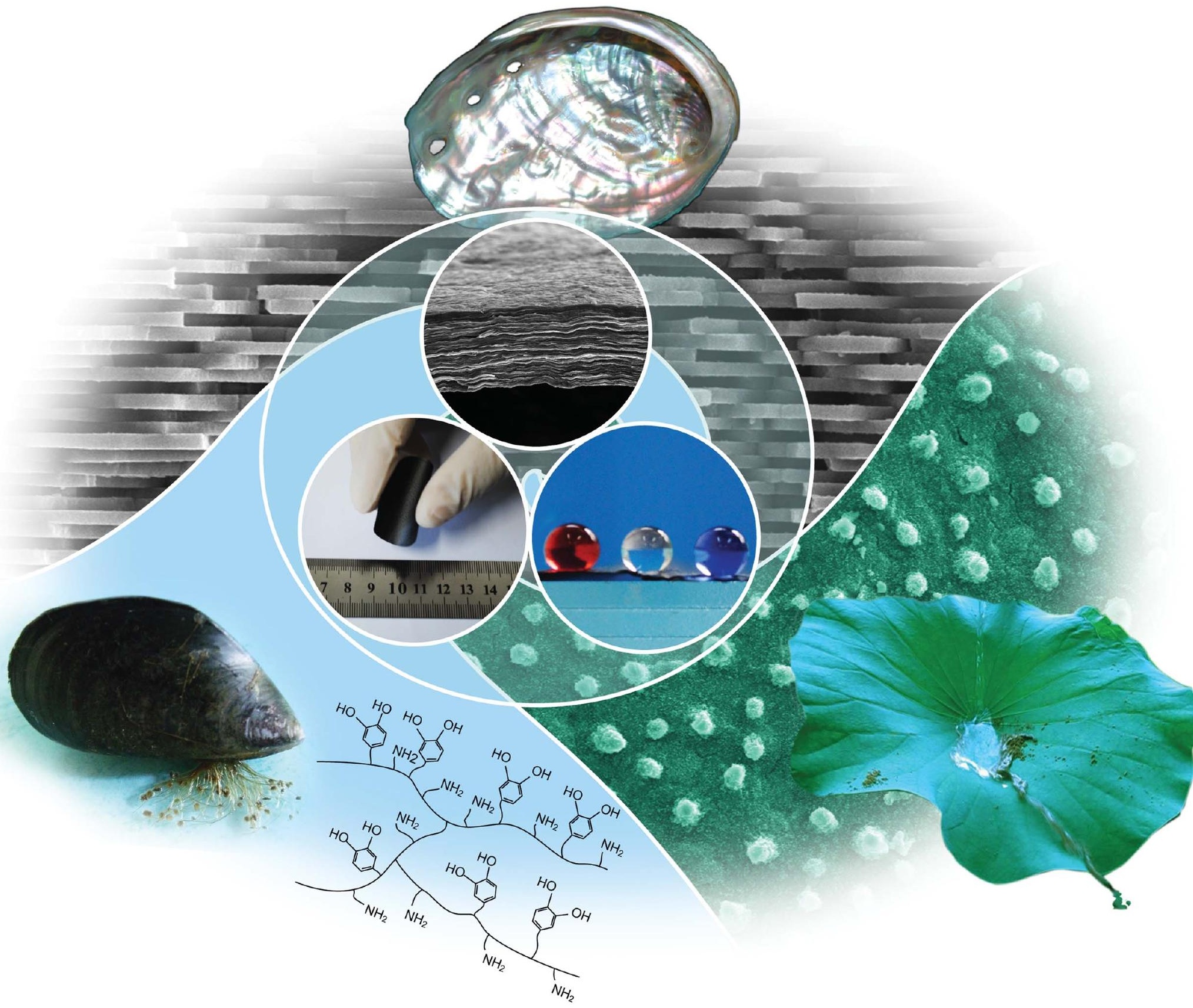

ISSN 2040-3364

PAPER

Yang, Liu et al.

Fusion of nacre, mussel, and lotus leaf: bio-inspired graphene composite

paper with multifunctional integration

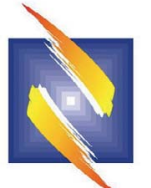

NCNST

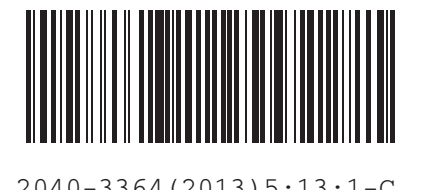




\title{
Fusion of nacre, mussel, and lotus leaf: bio-inspired graphene composite paper with multifunctional integration $\dagger$
}

\author{
Da Zhong, ${ }^{a}$ Qinglin Yang, ${ }^{* a}$ Lin Guo, ${ }^{a}$ Shixue Dou, ${ }^{b}$ Kesong Liu*ab and Lei Jiang ${ }^{\text {ac }}$ \\ Multifunctional integration is an inherent characteristic for biological materials with multiscale structures. \\ Learning from nature is an effective approach for scientists and engineers to construct multifunctional \\ materials. In nature, mollusks (abalone), mussels, and the lotus have evolved different and optimized \\ solutions to survive. Here, bio-inspired multifunctional graphene composite paper was fabricated in situ \\ through the fusion of the different biological solutions from nacre (brick-and-mortar structure), mussel \\ adhesive protein (adhesive property and reducing character), and the lotus leaf (self-cleaning effect). \\ Owing to the special properties (self-polymerization, reduction, and adhesion), dopamine could be \\ simultaneously used as a reducing agent for graphene oxide and as an adhesive, similar to the mortar \\ in nacre, to crosslink the adjacent graphene. The resultant nacre-like graphene paper exhibited stable \\ superhydrophobicity, self-cleaning, anti-corrosion, and remarkable mechanical properties underwater.
}

Received 14th November 2012

Accepted 14th January 2013

DOI: $10.1039 / c 3 n r 33632 h$

www.rsc.org/nanoscale

\section{Introduction}

Since life is estimated to have appeared on the Earth, nature has gone through billions of years of evolution and has learned what is optimal by using ordinary compositions. In the last few decades, a great variety of biological materials have been investigated by scientists and engineers. It was found that multiscale structures from the molecular to the nanoscale, microscale, and macroscale are characteristic for biological materials, exhibiting inherent multifunctional integration..$^{1-3}$ Creating multifunctional materials is an eternal goal for human beings. Optimized biological solutions provide a source of inspiration for scientists to design rationally and to construct reproducibly multiscale structures for multifunctional integration..$^{4-8}$

In nature, nacre, glass sponges, teeth, bones, and other biomaterials have evolved different solutions to overcome the brittleness of their building materials. ${ }^{9-11}$ Among the variety of biological materials, nacre is one of most promising owing to its superior mechanical strength and toughness (Fig. 1a). The distinctive mechanical properties can be attributed to a highly regular brick-and-mortar arrangement of inorganic and organic

${ }^{a}$ Key Laboratory of Bio-Inspired Smart Interfacial Science and Technology of Ministry of Education, School of Chemistry and Environment, Beihang University, Beijing 100191, PR China.E-mail: liuks@buaa.edu.cn; yangql@buaa.edu.cn

${ }^{b}$ Institute for Superconducting and Electronic Materials, University of Wollongong, Innovation Campus, Squires Way, North Wollongong, NSW 2500, Australia

'Beijing National Laboratory for Molecular Sciences, Key Laboratory of Organic Solids, Institute of Chemistry, Chinese Academy of Sciences, Beijing 100190, PR China

$\dagger$ Electronic supplementary information (ESI) available. See DOI: $10.1039 / \mathrm{c} 3 \mathrm{nr} 33632 \mathrm{~h}$ elements, consisting of predominately brittle calcium carbonate and a few percent of biomacromolecules (Fig. 1b and c). For nacre, the brick-and-mortar structure results in a 1000-fold increase in toughness over its constituent materials. The structure-function harmony of nacre has attracted significant attention from scientists to construct bio-inspired materials and to reproduce nature's achievements. ${ }^{12}$

In the sea, mussels, the notorious underwater fouling organisms, can attach to a variety of natural or synthetic substrates in high binding strength under wet conditions (Fig. 1d). The presence of 3,4-dihydroxy-phenylalanine (DOPA), a catecholic amino acid in secreted mussel adhesive proteins, is responsible for the strong underwater adhesion (Fig. 1e). ${ }^{13-15}$ DOPA demonstrates high affinity for chemically distinct substrates via covalent or noncovalent interactions. Dopamine contains both side chain functionalities of DOPA and lysine, which resembles mussel adhesive proteins (Fig. 1f). ${ }^{16-18}$ At a weak alkaline $\mathrm{pH}$, dopamine will undergo self-polymerization to form an adherent polydopamine coating on a large variety of substrates, demonstrating strong interfacial adhesion strength. ${ }^{16}$ In addition to its fascinating adhesive properties, dopamine also possesses a remarkable reducing character, which can be used as an eco-friendly and effective reducing agent to fabricate polymer nanocomposites. ${ }^{19}$

In Asia, the sacred lotus (Nelumbo nucifera) has been a symbol of purity for over 2000 years. Water droplets can roll freely in all directions and then pick up dirt particles on lotus leaves, resulting in the so-called self-cleaning effect or lotus effect (Fig. 1g). Lotus leaf surfaces possess randomly distributed micropapillae covered by branch-like nanostructures (Fig. $1 \mathrm{~h}$ and i). The cooperation of surface multiscale structures and 

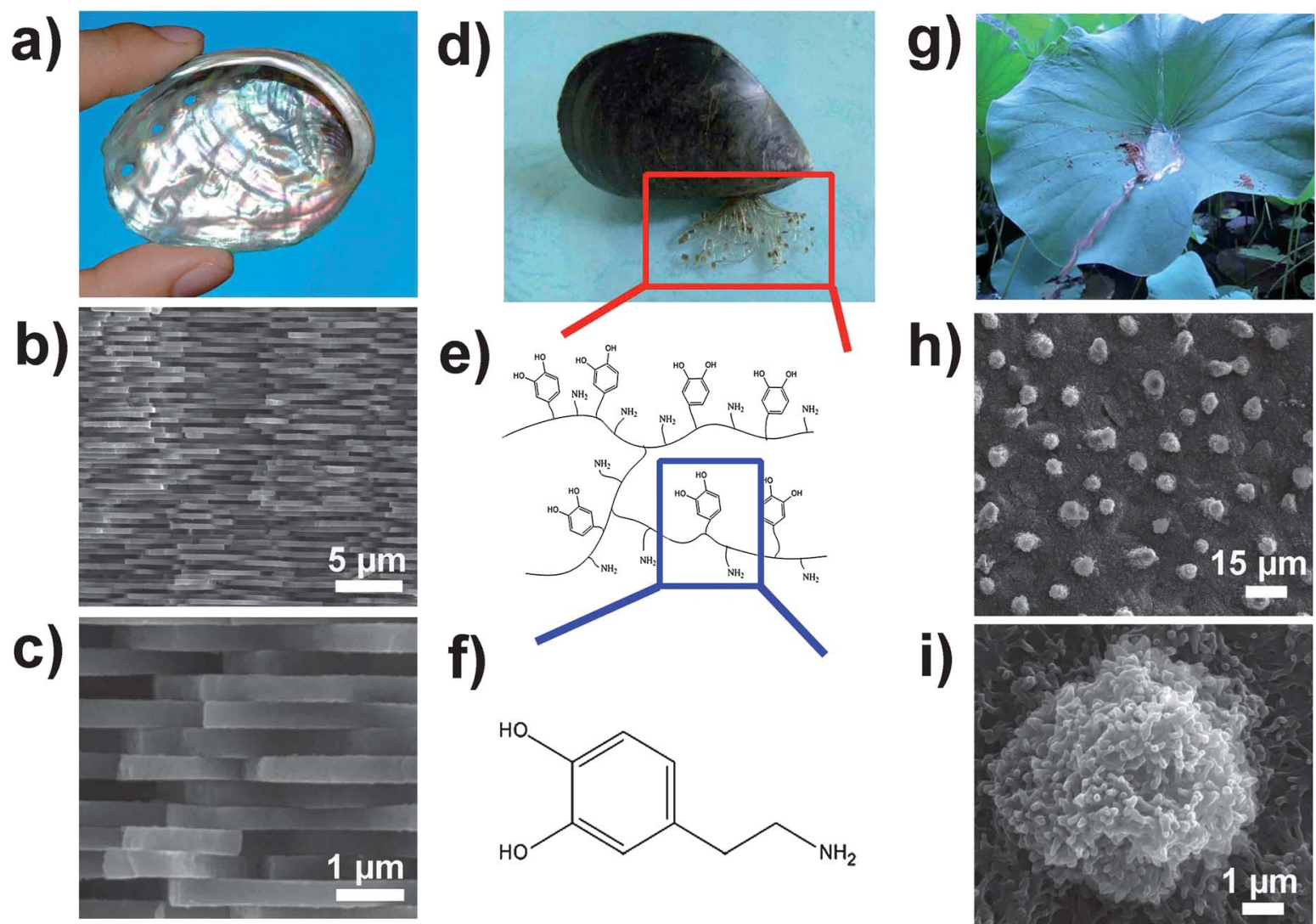

i)

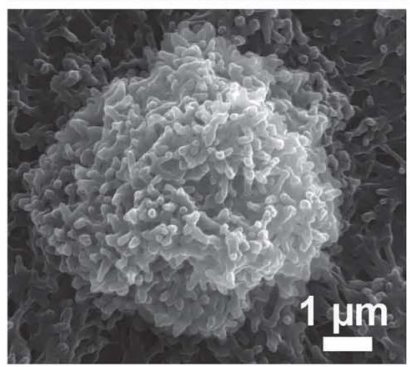

Fig. 1 (a) Digital image of the inner nacreous layer of the abalone shell. (b and c) ESEM images of a fracture surface in nacre at different magnification, exhibiting the layered bricks-and-mortar structure. (d) Photograph of a mussel attached to a glass substrate, showing the byssal threads and adhesive pads. (e) Simplified molecular representation of the mussel adhesive protein containing DOPA. (f) Dopamine contains both amine and catechol functional groups found in Mefp-5. (g) Digital photograph of a lotus leaf, where water droplets roll easily across the leaf surface and pick up dirt particles, demonstrating the self-cleaning effect. (h and i) ESEM images of the lotus leaf at different magnification, showing the micropapillae in a random distribution $(h)$ and cilium-like nanostructures superimposed on top of the micropapillae (i).

a)

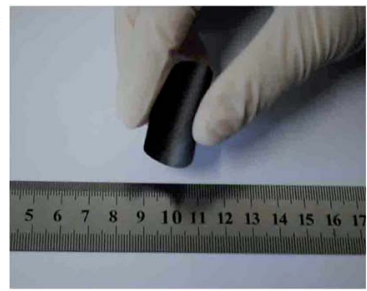

b)

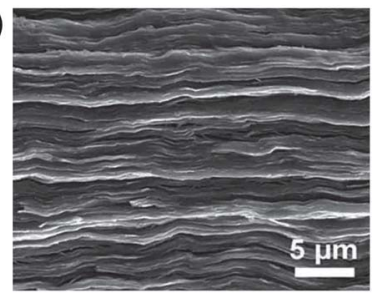

Fig. 2 Morphology and structure of the graphene composite paper. (a) Digital photograph of the folded graphene composite paper, exhibiting macroscopic flexibility and a slightly shiny surface. (b) High resolution ESEM side-view image of $25 \mu \mathrm{m}$ thick GC paper, showing a pronounced nacre-like layered structure.

hydrophobic epicuticular wax confers a high water contact angle and a small sliding angle. During the past few decades, many different synthesis strategies have been developed to design and fabricate superhydrophobic self-cleaning surfaces. ${ }^{20-22}$ Nowadays, a great variety of self-cleaning surfaces have been commercialized. ${ }^{23}$

Recently, the design and fabrication of $2 \mathrm{D}$ or $3 \mathrm{D}$ multifunctional materials has attracted wide scientific attention, which has become an increasingly hot research topic. ${ }^{24-26}$ For example, 3D multifunctional graphene-based hydrogels and aerogels have been fabricated on a large scale through a metal ion induced self-assembly approach. ${ }^{27}$ Graphene was considered as a rapidly rising star material and attracted a great deal of attention owing to its excellent mechanical, electrical, thermal, and optical properties. ${ }^{28-31} \mathrm{~A}$ key topic in the research and applications of graphene is the reduction of graphene oxide (GO). Nowadays, many different synthesis strategies have been developed to produce graphene through the reduction of GO ${ }^{32-35}$ Among the wide variety of synthesis strategies, chemical reduction of GO using dopamine reagent is one of the most promising. ${ }^{36-41}$ In such a process, dopamine acts simultaneously as a reducing agent in situ for GO and as a capping agent to stabilize and decorate the resultant graphene surface for further functionalization, owing to its fascinating properties (reduction, self-polymerization, and adhesion). Furthermore, dopamine can absorb on the graphene surface due to its excellent affinity. ${ }^{42}$ During the past few decades, graphene has been well-investigated and much work has been devoted to the synthesis, structures, properties, and possible applications of graphene..$^{43-45}$ Less work has been directed to the surface wetting behavior of graphene, although the wetting behavior of solid surfaces is a very important aspect of materials and can strongly affect their properties. ${ }^{46}$ Recently, the wettability of 


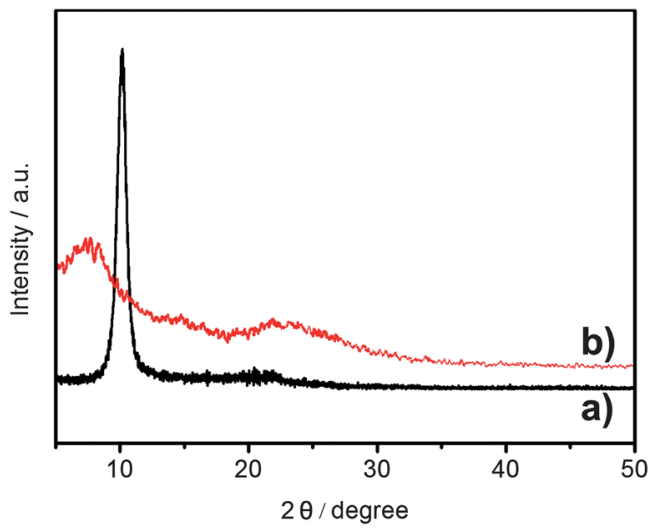

Fig. 3 XRD patterns of graphene oxide (a) and graphene composite paper (b). The disappearance of the sharp diffraction peak $\left(2 \theta=10.3^{\circ}\right)$ and a new broad peak $\left(2 \theta=23.6^{\circ}\right)$ confirmed the reduction of graphene oxide by polydopamine.

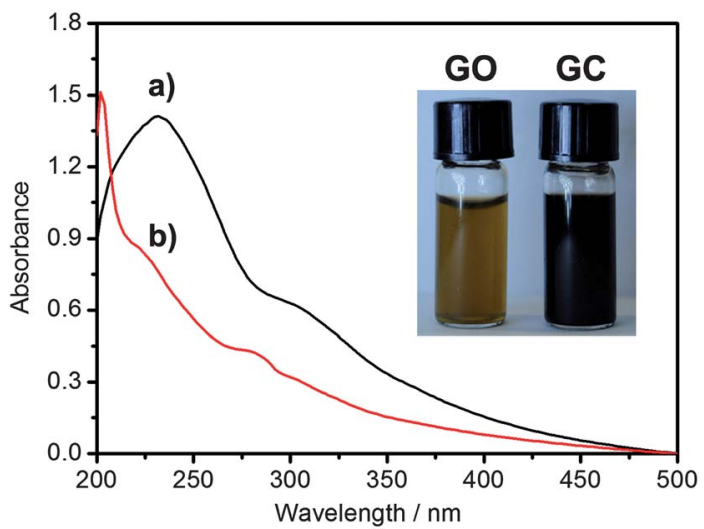

Fig. 4 UV-vis absorption spectra of the aqueous dispersion of graphene oxide and graphene composite. The new peak at $280 \mathrm{~nm}$ indicated the presence of polydopamine in the graphene composite. Insets are typical images of aqueous suspensions of graphene oxide (left) and graphene composite (right) showing the light brown and black color, respectively, demonstrating the reduction of graphene oxide to graphene.

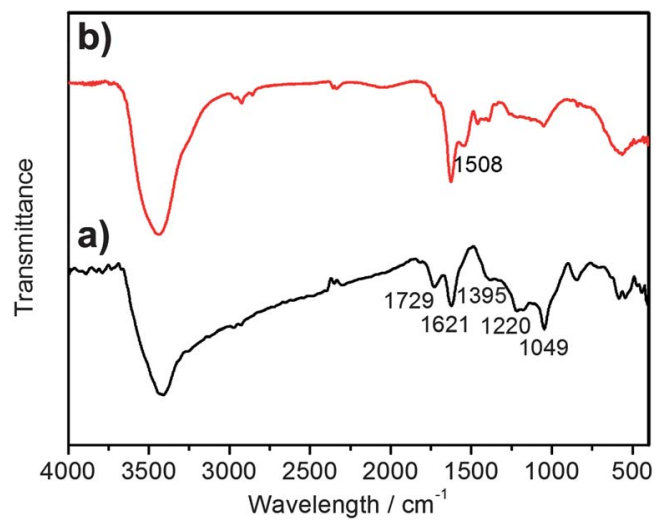

Fig. 5 FT-IR spectra of graphene oxide (a) and graphene composite (b). The disappearance of a characteristic peak of graphene oxide at $1729 \mathrm{~cm}^{-1}(C=O)$ and the new peak at $1508 \mathrm{~cm}^{-1}(\mathrm{~N}-\mathrm{H})$ strongly demonstrated the reduction of graphene oxide and the presence of polydopamine in graphene composite paper. graphene films could be tuned from superhydrophobic to superhydrophilic by simply controlling the relative proportion of acetone and water in the solvent, which is important to expand the applications of graphene. ${ }^{47}$

In the present work, we demonstrate an environmentally friendly approach for the in situ fabrication of bio-inspired graphene composite (GC) paper with multifunctional integration through the fusion of seemingly distinct concepts of nacre (brick-and-mortar structure), mussel adhesive protein (adhesive property and reducing character), and the lotus leaf (selfcleaning effect). The amazing properties (self-polymerization, reduction, and adhesion) of dopamine make it possible to use it simultaneously as a reducing agent for GO and as an adhesive similar to the mortar in nacre to crosslink lamellar graphene in situ. Although nacre-like graphene composites have been fabricated using different approaches, ${ }^{48-50}$ in our case, the resultant paper-like GC demonstrates stable superhydrophobicity, self-cleaning, and remarkable mechanical properties underwater, exhibiting multifunctional integration.

\section{Experimental}

Fabrication of multifunctional graphene composite paper

In a typical procedure, graphite oxide was synthesized using graphite (200 mesh, Alfa Aesar) by a modified Hummers method. ${ }^{51}$ Individual graphene oxide (GO) sheets were exfoliated by dissolving graphite oxide $(36 \mathrm{mg})$ in deionized water $(80$ $\mathrm{mL})$ with the help of ultrasound. Then, trizma base $(121 \mathrm{mg}$, Aldrich) was put into the suspension and the $\mathrm{pH}$ was adjusted to 8.5 using hydrochloric acid. After addition of dopamine hydrochloride (18 mg, Alfa Aesar), the colloidal suspension was adjusted to $100 \mathrm{~mL}$. The mixed suspension was further stirred at room temperature for $4 \mathrm{~h}$, resulting in the formation of graphene-polydopamine composites. Graphene composite (GC) paper was fabricated by vacuum filtration of dialyzed graphene dispersions through a cellulose acetate membrane filter ( $0.22 \mu \mathrm{m}$ pore size, $50 \mathrm{~mm}$ diameter), followed by air drying and peeling from the filter. For comparison, the equivalent pristine GO paper was fabricated by filtering a GO dispersion similar to the above described approach without the addition of dopamine. The surface functionalization of GC paper was performed in accordance with the literature procedure. ${ }^{52}$ Polystyrene granules (1.0 g, Aldrich), hydrophobic fumed silica nanoparticles ( $0.8 \mathrm{~g}$, Evonik Degussa Co.), and chloroform (30 mL, Aldrich) were mixed and magnetically stirred for $0.5 \mathrm{~h}$ in a closed bottle. Superhydrophobic GC paper was produced by a dip-coating method at a speed of $c a .0 .5 \mathrm{~cm} \mathrm{~s}^{-1}$.

\section{Characterization}

Environmental scanning electron microscopy (ESEM, FEI Quanta 250 FEG) was used to characterize the microscale structure of the sample at an acceleration voltage of $10 \mathrm{kV}$. Ultraviolet-visible (UV-vis) spectra were obtained using a Cintra 10e spectrophotometer (GBC Scientific Equipment Pty Ltd, Australia). The aqueous suspension of GO and GC was used as the UV-vis sample, and $10 \mathrm{mM}$ Tris-Cl solution $(\mathrm{pH}=8.5)$ was 

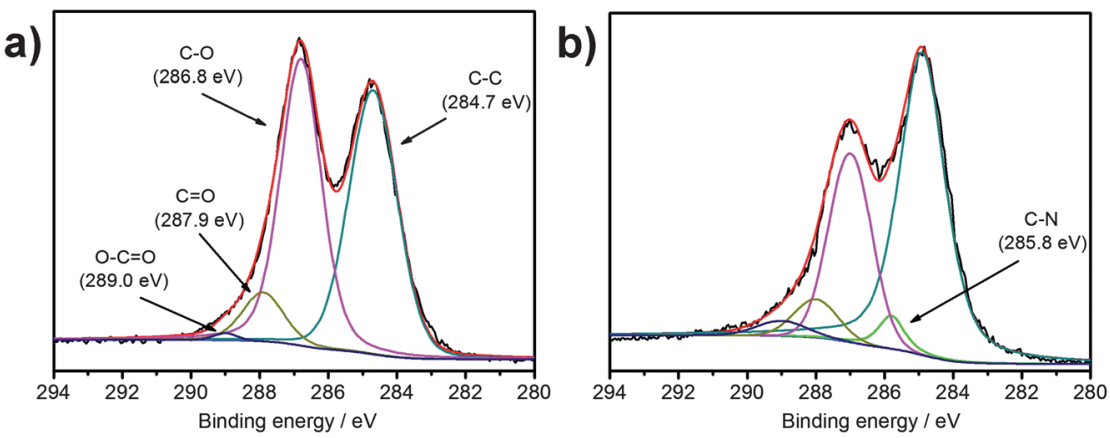

Fig. 6 C1s XPS spectra of graphene oxide (a) and graphene composite (b). The decrease of peak intensities at 284-290 eV and the appearance of a new C-N peak at $285.8 \mathrm{eV}$ demonstrated the reduction of $\mathrm{GO}$ by dopamine and the presence of polydopamine coating in the GC paper.

used as the reference. X-Ray diffraction (XRD) analyses were carried out on an X-ray diffractometer (D/MAX-1200, Rigaku Denki Co. Ltd, Japan). Fourier transform infrared (FT-IR) spectra of the samples were recorded on an Avatar 360 spectrophotometer (Thermo Nicolet, USA). X-Ray photoelectron spectroscopy (XPS) data were obtained with an ESCALab220i-XL electron spectrometer from VG Scientific using $300 \mathrm{~W}$ Al K $\alpha$ radiation. The mechanical property test was conducted with a universal testing machine (AGS-X, Shimadzu Co. Ltd, Japan). The samples were mounted using film tension clamps with a clamp compliance of about $0.2 \mu \mathrm{m} \mathrm{N}{ }^{-1}$. All tensile tests were performed in controlled force mode with a preload of $0.01 \mathrm{~N}$ and the force was loaded with a force ramp rate of $0.05 \mathrm{~mm}$ $\min ^{-1}$. Water contact angles were measured on an OCA20 instrument (Dataphysics, Germany) contact angle system at room temperature. The average water contact angle was obtained by measuring more than five different positions of the same sample.

\section{Results and discussion}

A photograph of the GC paper fabricated by vacuum filtration of the as-reduced dispersion through a cellulose acetate membrane is shown in Fig. 2a. The GC paper is uniform and possesses a black color. Furthermore, even after bending, the paper exhibits high and macroscopic flexibility, which is very important for the practical application of GC. The entire crosssection of the GC paper is characterized by ESEM. The highresolution ESEM image clearly shows that the GC paper has well-defined layered architecture with strictly planar orientation, which resembles the brick-and-mortar structure of nacre. The total thickness of GC paper is about $25 \mu \mathrm{m}$. The size and thickness of the paper can be easily adjusted by the size of the membrane filter and the volume/concentration of GC dispersion, respectively.

XRD pattern shown in Fig. 3 presents a typical pattern for GO and GC specimen..$^{53,54}$ The layer-to-layer distance ( $d$-spacing) is
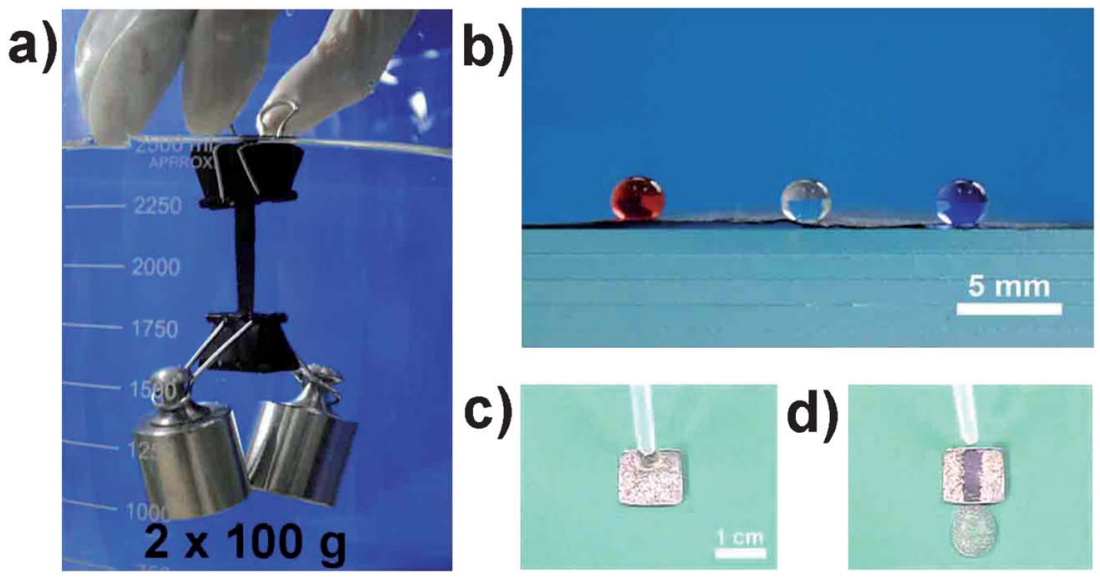

Fig. 7 (a) Photograph of more than $200 \mathrm{~g}$ in weight (weights, clamp) supported by the graphene composite paper (thickness $=25 \mu \mathrm{m}$ and width $=5 \mathrm{~mm}$ ) with nacrelike brick-and-mortar structures, exhibiting considerable mechanical properties under water. (b) Typical optical image of aqueous hydrochloric acid (left, $\mathrm{pH}=1$ ), $\mathrm{NaCl}$ (middle, $\mathrm{pH}=7$ ), and $\mathrm{NaOH}$ (right, $\mathrm{pH}=14$ ) droplets of spherical shape on the graphene composite paper, demonstrating stable superhydrophobicity towards different corrosive solutions. ( $c$ and d) Demonstration of the self-cleaning ability of the superhydrophobic graphene composite paper. Glass powder was spread on graphene composite paper. Contaminants can be efficiently removed from the surface of graphene composite paper using a water droplet, showing the lotus leaf-like self-cleaning effect. 
a)

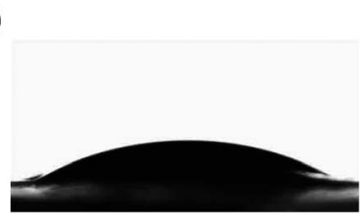

b)

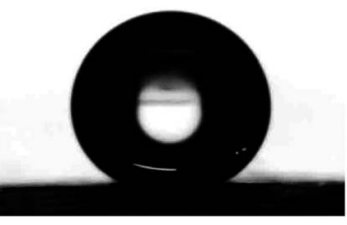

Fig. 8 Water static contact angle images of graphene composite paper modified without (a) and with (b) hydrophobic fumed silica. After modification, the original graphene composite paper was transformed from hydrophilicity (about $30^{\circ}$ ) to superhydrophobicity (about $153^{\circ}$ ).

about $0.86 \mathrm{~nm}$. However, after reduction with dopamine, the sharp diffraction peak in GO has become weak. A new broad diffraction peak at $23.6^{\circ}(d$-spacing $=0.37 \mathrm{~nm})$ can be observed for the resultant GC paper, indicating GO is indeed reduced by dopamine to some extent. ${ }^{37-39}$ Fig. 4 illustrates the UV-vis absorption spectra of GO and GC in the Tris-Cl solution. For $\mathrm{GO}$, a characteristic absorbance peak at about $230 \mathrm{~nm}$ and a shoulder peak at around 290-300 nm can be observed (Fig. 4a), which can be indexed as the $\pi \rightarrow \pi^{*}$ of $\mathrm{C}=\mathrm{C}$ and $\mathrm{n} \rightarrow \pi^{*}$ transition of the carbonyl groups. ${ }^{36}$ The UV-vis absorption spectra shown in Fig. 4b demonstrate a successful reduction of GO to graphene by dopamine. Furthermore, a new peak at 280 $\mathrm{nm}$ was detected, which is a typical absorption of catechols in polydopamine. ${ }^{40}$ A similar behavior can be observed in the simultaneous reduction and surface functionalization of graphene oxide utilizing mussel-inspired poly(norepinephrine)containing catechols. ${ }^{55}$ Insets of Fig. 4 present the digital images of the GO and GC suspensions. After the addition of dopamine, the color of the GO suspension changed from light brown to black, implying the reduction of GO to graphene. ${ }^{56,57}$ Furthermore, the obtained GC samples demonstrated excellent aqueous stability. No sediment was observed even after the GC dispersion was stored for more than three months. The high aqueous stability of GC can be attributed to the surface decoration of polydopamine.

The simultaneous reduction of GO to GC and functionalization of GC by dopamine were further demonstrated by FT-IR and XPS spectra, exhibited in Fig. 5 and 6, respectively. The FTIR spectrum of GO clearly shows the following characteristic peaks (Fig. 5a): the $\mathrm{C}=\mathrm{O}$ stretching vibration peak of carboxyl groups at $1729 \mathrm{~cm}^{-1}$; the aromatic $\mathrm{C}=\mathrm{C}$ stretching vibration peak at $1621 \mathrm{~cm}^{-1}$; the $\mathrm{O}-\mathrm{H}$ deformation vibration peak at $1395 \mathrm{~cm}^{-1}$; the $\mathrm{C}-\mathrm{OH}$ stretching peak at $1220 \mathrm{~cm}^{-1}$; and the C-O stretching peak at $1049 \mathrm{~cm}^{-1} \cdot$. $^{39-41}$ The disappearance of the typical $\mathrm{C}=\mathrm{O}$ peak at $1729 \mathrm{~cm}^{-1}$ compared to original GO strongly demonstrated the reduction of GO to graphene by the reaction between carboxylic acid groups and amine groups (Fig. 5b). The new peak at $1508 \mathrm{~cm}^{-1}$ can be assigned to the $\mathrm{C}-\mathrm{N}$ shearing vibration of the amide group in polydopamine, indicating the presence of polydopamine in GC paper. ${ }^{40}$

The samples of GO and GC were also characterized by XPS. The C1s spectrum of GO can be divided into four components with binding energies at about 284.7, 286.8, 287.9 and $289.0 \mathrm{eV}$, corresponding to carbon atoms in $\mathrm{C}-\mathrm{C}, \mathrm{C}-\mathrm{O}, \mathrm{C}=\mathrm{O}$ and $\mathrm{O}-\mathrm{C}=\mathrm{O}$ species, respectively (Fig. 6a). However, the $\mathrm{C}-\mathrm{O}$ peak intensity at $286.8 \mathrm{eV}$ of GC is obviously weaker than that of GO, (Fig. 6b), implying the reduction of GO by dopamine. Furthermore, for the GC sample, the appearance of a new C-N peak at the binding energy at about $285.8 \mathrm{eV}$ strongly demonstrated the presence of the polydopamine coating in the multifunctional GC paper. ${ }^{37,38,41}$ The above ESEM, XRD, UV-vis, FT-IR, and XPS observations proved that GO is indeed reduced by dopamine and polydopamine is present in the GC paper.

Research indicated that the nacre-like brick-and-mortar structure provides an avenue for the construction of advanced materials with special mechanical properties. Brinson et al. presented GO films with lamellar structures that can be used as load-bearing structural materials in air. ${ }^{58}$ However, for some applications, graphene may need to work under water and retain its impressive mechanical properties. To demonstrate the macroscale application of flexible and superhydrophobic GC paper in a water environment, two weights were loaded on the GC paper ( $25 \mu \mathrm{m}$ in thickness). An overall mass of more than $200 \mathrm{~g}$ was calculated for GC paper shown in Fig. 7a, showing considerable mechanical properties under water. The mechanical properties of the GC paper were also measured using the universal testing machine. The stress-strain curve of the GC

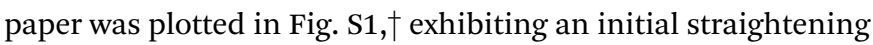
region followed by an "linear region" (this region contains an

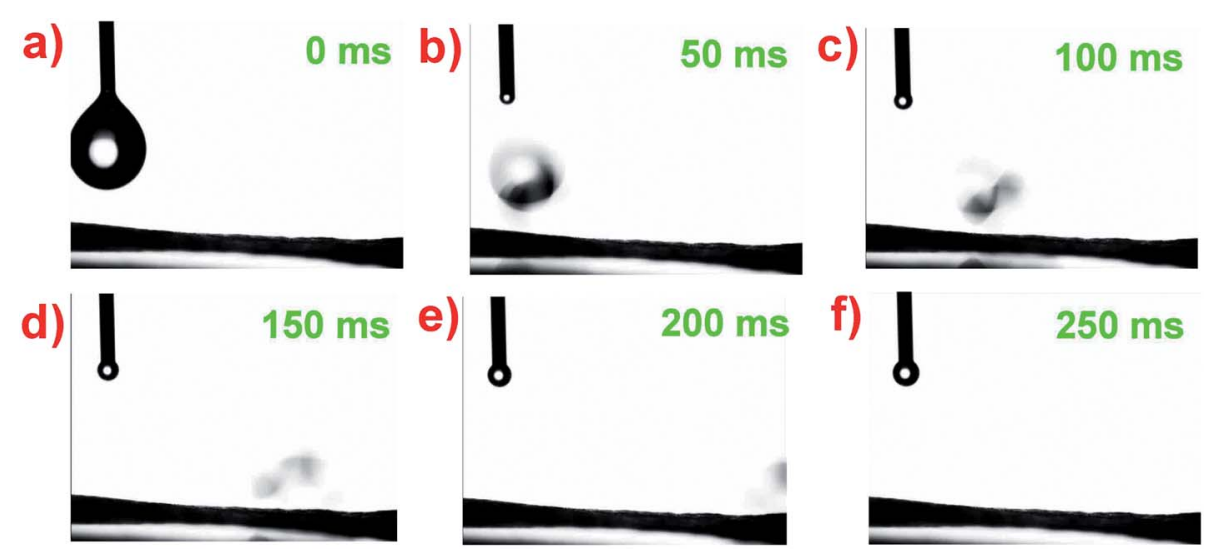

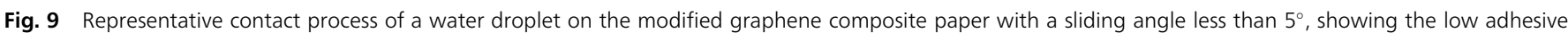
superhydrophobicity. 
elastic region and a plastic region but it is almost linear, therefore we here refer to it as the linear region). ${ }^{59}$ The obtained GC paper has a Young's modulus of $13.9 \mathrm{GPa}$. The value of the ultimate tensile strength of the GC paper can reach 43.1 MPa, which is enough to support two 100 gram weights in water.

Wettability is a very important characteristic of solid surfaces, which is generally governed by both surface geometric structures and surface chemical compositions. For the original GC paper, the water static contact angle is less than $30^{\circ}$ (Fig. 8a), exhibiting intrinsic hydrophilicity due to the presence of polydopamine on the GC surfaces. ${ }^{16}$ After modification with low surface-free-energy silica nanoparticles, GC paper demonstrates superhydrophobicity with a water static contact angle larger than $153^{\circ}$ (Fig. 8b). The generation of superhydrophobicity can be attributed the cooperation of surface hierarchical structures and low surface energy silica. ${ }^{52}$ In addition to the water repellency, the modified GC paper also exhibited repellency towards corrosive liquids, such as acidic, basic, and some aqueous salt solutions. The static contact angle is almost unchanged over a wide range of $\mathrm{pH}$ values from 1 to 14 , demonstrating good stability. Fig. $7 \mathrm{~b}$ shows the representative digital image of acidic (left, $\mathrm{pH}=1$ ), salt (middle, $\mathrm{pH}=7$ ), and basic (right, $\mathrm{pH}=14$ ) droplets on the GC paper surface. All these aqueous solution droplets with spherical shape were located uniformly on the modified GC paper, exhibiting stable superhydrophobicity even towards many corrosive solutions. Furthermore, the water droplet cannot stick to the GC paper surface and the sliding angle is lower than $5^{\circ}$, allowing water droplets to roll off quite easily. This can be directly confirmed by the evolving contact process of a water droplet on GC paper (Fig. 9), where a water droplet slides on the surface quite quickly in a very short time (about $200 \mathrm{~ms}$ ), demonstrating low adhesive superhydrophobic properties. Functional surfaces simultaneously possessing a high static water contact angle and a low sliding angle have an important application in the field of self-cleaning. To investigate the self-cleaning ability, the superhydrophobic GC paper was contaminated with glass powder (Fig. 7c). These dirt particles can be readily removed from the GC surface by rolling a water droplet (Fig. 7d), exhibiting the lotus leaf-like selfcleaning effect.

\section{Conclusions}

In conclusion, bio-inspired multifunctional GC paper has been fabricated in situ through the fusion of the seemingly distinct concepts of nacre (brick-and-mortar structure), mussel adhesive protein (adhesive property and reducing character), and the lotus leaf (self-cleaning effect). Under water, GC paper $(25 \mu \mathrm{m}$ in thickness) with nacre-like layered brick-and-mortar multiscale structures can support more than $200 \mathrm{~g}$ of mass, demonstrating considerable mechanical properties. In addition, GC paper exhibited superior repellency towards water, even including corrosive liquids (such as acidic, basic, and some aqueous salt solutions) and the lotus leaf-like self-cleaning effect. Superhydrophobic GC paper with well-defined layered structures and the self-cleaning effect should extend the practical applications of graphene in a wide range of different fields. We anticipate this facile method can be widely adopted for the fabrication of other bio-inspired graphene-based composites with multifunctional integration.

\section{Acknowledgements}

We appreciate the financial support of the National Basic Research Program of China (2010CB934700, 2013CB933003), the National Natural Science Foundation of China (21273016, 21001013), the Program for New Century Excellent Talents in University, Beijing Natural Science Foundation (2122035), the Key Research Program of the Chinese Academy of Sciences (KJZD-EW-M01), and the Fundamental Research Funds for the Central Universities.

\section{References}

1 K. Liu and L. Jiang, Nano Today, 2011, 6, 155-175.

2 J. Aizenberg and P. Fratzl, Adv. Mater., 2009, 21, 387-388.

3 K. Liu and L. Jiang, ACS Nano, 2011, 5, 6786-6790.

4 A. Grinthal, S. H. Kang, A. K. Epstein, M. Aizenberg, M. Khan and J. Aizenberg, Nano Today, 2012, 7, 35-52.

5 B. J. Kim, Y. S. Choi and H. J. Cha, Angew. Chem., Int. Ed., 2012, 51, 675-678.

6 P. Podsiadlo, Z. Liu, D. Paterson, P. B. Messersmith and N. A. Kotov, Adv. Mater., 2007, 19, 949-955.

7 Y. F. Li, J. H. Zhang and B. Yang, Nano Today, 2010, 5, 117127.

8 K. Liu and L. Jiang, Nanoscale, 2011, 3, 825-838.

9 P. Fratzl and R. Weinkamer, Prog. Mater. Sci., 2007, 52, 12631334.

10 M. A. Meyers, P. Y. Chen, A. Y. M. Lin and Y. Seki, Prog. Mater. Sci., 2008, 53, 1-206.

11 J. Aizenberg, J. C. Weaver, M. S. Thanawala, V. C. Sundar, D. E. Morse and P. Fratzl, Science, 2005, 309, 275-278.

12 Z. Y. Tang, N. A. Kotov, S. Magonov and B. Ozturk, Nat. Mater., 2003, 2, 413-418.

13 B. P. Lee, P. B. Messersmith, J. N. Israelachvili and J. H. Waite, Annu. Rev. Mater. Res., 2011, 41, 99-132.

14 J. H. Waite and M. L. Tanzer, Science, 1981, 212, 1038-1040. 15 Q. Lin, D. Gourdon, C. J. Sun, N. Holten-Andersen, T. H. Anderson, J. H. Waite and J. N. Israelachvili, Proc. Natl. Acad. Sci. U. S. A., 2007, 104, 3782-3786.

16 H. Lee, S. M. Dellatore, W. M. Miller and P. B. Messersmith, Science, 2007, 318, 426-430.

17 J. H. Waite, Nat. Mater., 2008, 7, 8-9.

18 Q. Ye, F. Zhou and W. Liu, Chem. Soc. Rev., 2011, 40, 42444258.

19 Y. Fu, P. Li, Q. Xie, X. Xu, L. Lei, C. Chen, C. Zou, W. Deng and S. Yao, Adv. Funct. Mater., 2009, 19, 1784-1791.

20 J. Zhu, C. M. Hsu, Z. F. Yu, S. H. Fan and Y. Cui, Nano Lett., 2010, 10, 1979-1984.

21 K. Liu, X. Yao and L. Jiang, Chem. Soc. Rev., 2010, 39, 32403255.

22 T. S. Wong, S. H. Kang, S. K. Y. Tang, E. J. Smythe, B. D. Hatton, A. Grinthal and J. Aizenberg, Nature, 2011, 477, 443-447. 
23 K. Liu and L. Jiang, Annu. Rev. Mater. Res., 2012, 42, 231-263. 24 H. W. Liang, Q. F. Guan, L. F. Chen, Z. Zhu, W. J. Zhang and S. H. Yu, Angew. Chem., Int. Ed., 2012, 51, 5101-5105.

25 H. B. Yao, L. B. Mao, Y. X. Yan, H. P. Cong, X. Lei and S. H. Yu, ACS Nano, 2012, 6, 8250-8260.

26 J. L. Vickery, A. J. Patil and S. Mann, Adv. Mater., 2009, 21, 2180.

27 H. P. Cong, X. C. Ren, P. Wang and S. H. Yu, ACS Nano, 2012, 6, 2693-2703.

28 A. K. Geim, Angew. Chem., Int. Ed., 2011, 50, 6966-6985.

29 K. S. Novoselov, Angew. Chem., Int. Ed., 2011, 50, 6986-7002.

30 L. P. Biro, P. Nemes-Incze and P. Lambin, Nanoscale, 2012, 4, 1824-1839.

31 N. Zhang, Y. H. Zhang and Y. J. Xu, Nanoscale, 2012, 4, 57925813.

32 S. F. Pei and H. M. Cheng, Carbon, 2012, 50, 3210-3228.

33 H. Bai, C. Li and G. Shi, Adv. Mater., 2011, 23, 1089-1115.

34 S. Mao, H. H. Pu and J. H. Chen, RSC Adv., 2012, 2, 26432662.

35 J. Kang, D. Shin, S. Bae and B. H. Hong, Nanoscale, 2012, 4, 5527-5537.

36 Z. T. Luo, Y. Lu, L. A. Somers and A. T. C. Johnson, J. Am. Chem. Soc., 2009, 131, 898-899.

37 L. Q. Xu, W. J. Yang, K. G. Neoh, E. T. Kang and G. D. Fu, Macromolecules, 2010, 43, 8336-8339.

38 L. Yang, W. A. Yee, S. L. Phua, J. Kong, H. Ding, J. W. Cheah and X. Lu, RSC Adv., 2012, 2, 2208-2210.

39 Y. Mi, Z. Wang, X. Liu, S. Yang, H. Wang, J. Ou, Z. Li and J. Wang, J. Mater. Chem., 2012, 22, 8036-8042.

40 H. Liu, P. Xi, G. Xie, Y. Shi, F. Hou, L. Huang, F. Chen, Z. Zeng, C. Shao and J. Wang, J. Phys. Chem. C, 2012, 116, 3334-3341.

41 I. Kaminska, M. R. Das, Y. Coffinier, J. Niedziolka-Jonsson, J. Sobczak, P. Woisel, J. Lyskawa, M. Opallo, R. Boukherroub and S. Szunerits, ACS Appl. Mater. Interfaces, 2012, 4, 1016-1020.

42 H. L. Guo, X. F. Wang, Q. Y. Qian, F. B. Wang and X. H. Xia, ACS Nano, 2009, 3, 2653-2659.
43 D. S. Hecht, L. B. Hu and G. Irvin, Adv. Mater., 2011, 23, 1482-1513.

44 A. A. Balandin, S. Ghosh, W. Bao, I. Calizo, D. Teweldebrhan, F. Miao and C. N. Lau, Nano Lett., 2008, 8, 902-907.

45 M. Lafkioti, B. Krauss, T. Lohmann, U. Zschieschang, H. Klauk, K. von Klitzing and J. H. Smet, Nano Lett., 2010, 10, 1149-1153.

46 J. Rafiee, X. Mi, H. Gullapalli, A. V. Thomas, F. Yavari, Y. F. Shi, P. M. Ajayan and N. A. Koratkar, Nat. Mater., 2012, 11, 217-222.

47 J. Rafiee, M. A. Rafiee, Z. Z. Yu and N. Koratkar, Adv. Mater., 2010, 22, 2151-2154.

48 X. Wang, H. Bai, Z. Yao, A. Liu and G. Shi, J. Mater. Chem., 2010, 20, 9032-9036.

49 Y. Q. Li, T. Yu, T. Y. Yang, L. X. Zheng and K. Liao, Adv. Mater., 2012, 24, 3426-3431.

50 Y. Zhang, Q. Chen, Z. Jin, E. Kim and H. Sun, Nanoscale, 2012, 4, 4858-4869.

51 W. S. Hummers and R. E. Offeman, J. Am. Chem. Soc., 1958, 80, 1339.

52 B. Su, S. Wang, Y. Song and L. Jiang, Nano Res., 2010, 4, 266273.

53 D. A. Dikin, S. Stankovich, E. J. Zimney, R. D. Piner, G. Dommett, G. Evmenenko, S. T. Nguyen and R. Ruoff, Nature, 2007, 448, 457-460.

54 O. C. Compton, B. Jain, D. A. Dikin, A. Abouimrane, K. Amine and S. T. Nguyen, ACS Nano, 2011, 5, 43804391.

55 S. Kang, S. Park, D. Kim, S. Park, R. Ruoff and H. Lee, Adv. Funct. Mater., 2011, 21, 108-112.

56 D. Li, M. B. Muller, S. Gilje, R. B. Kaner and G. G. Wallace, Nat. Nanotechnol., 2008, 3, 101-105.

57 O. Akhavan, E. Ghaderi, S. Aghayee, Y. Fereydooni and A. Talebi, J. Mater. Chem., 2012, 22, 13773-13781.

58 Z. An, O. C. Compton, K. W. Putz, L. C. Brinson and S. T. Nguyen, Adv. Mater., 2011, 23, 3842-3846.

59 S. Park, D. A. Dikin, S. T. Nguyen and R. S. Ruoff, J. Phys. Chem. C, 2009, 113, 15801-15804. 